Enferm Bras 2019;18(2);176-83

https://doi.org/10.33233/eb.v18i2.1295

\title{
ARTIGO ORIGINAL \\ Conhecimentos, atitudes e práticas de responsáveis por crianças quilombolas acerca de primeiros socorros
}

lasmin Melo dos Santos*, Josely Bruce Santos, M.Sc. ${ }^{* \star}$, Lívia Pinheiro Pereira, M.Sc. ${ }^{* \star}$, Ridalva Dias Martins Felzemburgh, D.Sc. ${ }^{\star \star *}$, Saionara Costa do Sacramento*

${ }^{*}$ Enfermeira, Universidade Federal da Bahia, ${ }^{* *}$ Enfermeira, Mestre em Enfermagem, especialista em saúde da criança, Universidade Federal da Bahia, ${ }^{* * \star}$ Enfermeira, psicóloga, Doutora em Epidemiologia pela Fiocruz, Universidade Federal da Bahia

Recebido em 30 de outubro de 2017; aceito em 23 de outubro de 2018.

Endereço de correspondência: Josely Bruce Santos, Avenida Luís Viana, 661/1901, Torre Primavera, Paralela, 41730101 Salvador BA, E-mail: joselybruce3@gmail.com; lasmin Melo dos Santos: yasmin-melo2011@hotmail.com; Lívia Pinheiro Pereira: liv_pp@hotmail.com; Ridalva Dias Martins Felzemburgh: ridalva@gmail.com; Saionara Costa do Sacramento: saionaracosta2008@hotmail.com.br

\section{Resumo}

O objetivo desta pesquisa foi identificar os conhecimentos de pais e/ou responsáveis por crianças quilombolas acerca de medidas de primeiros socorros. Estudo descritivo exploratório com abordagem quantitativa, realizado através de aplicação de entrevistas domiciliares. A população foram pais e/ou responsáveis por crianças quilombolas entre 0 e 11 anos, 11 meses e 29 dias na comunidade quilombola em llha de Maré, Salvador/BA. Os resultados foram: alergias $68,9 \%$ buscam o serviço de saúde, diarreia $44,8 \%$ utilizam chás, $58,6 \%$ em casos de febre utilizam a automedicação, $24 \%$ em episódios de vômito buscam o serviço de saúde, $31,1 \%$ em casos de queimadura utilizam o resfriamento. Embora em alguns casos a comunidade procure o serviço de saúde, que podem ofertar resolutividade, a mesma encontra-se localizada à longa distância destes. Desta forma, torna-se necessário o estímulo de atividades educativas para que a comunidade tenha o acesso à saúde de forma integral.

Palavras-chave: comunidade, primeiros socorros, criança, acidentes, saúde.

\section{Abstract}

Knowledge, attitudes and practices of responsibles for quilombola children about first aid The objective of this research was to identify the knowledge of parents and/or responsible for quilombola children about first aid measures. Exploratory descriptive study with a quantitative approach, performed through the application of home interviews. The population was parents and/or responsible for quilombola children between 0 and 11 years, 11 months and 29 days in the quilombola community in Ilha de Maré, Salvador/BA. The results were: allergies $68.9 \%$ seek the health service, diarrhea $44.8 \%$ use teas, $58.6 \%$ in cases of fever use self-medication, $24 \%$ in episodes of vomiting seek the health service, $31.1 \%$ in burn cases use cooling. Although in some cases the community seeks the health service, which can offer resolution, it is located at long distance from them. In this way, it is necessary to stimulate educational activities so that the community has access to health services.

Key-words: community, first aid, child, accidents, health.

\section{Resumen}

Conocimientos, actitudes y prácticas de responsables por niños quilombolas acerca de primerosauxilios

El objetivo de esta investigación fue identificar los conocimientos de padres y/o responsables por niños quilombolas acerca de medidas de primeros auxilios. Estudio descriptivo exploratorio con abordaje cuantitativo, realizado a través de la aplicación de entrevistas domiciliarias. La población fue padres y/o responsables por niños quilombolas entre 0 a 11 años, 11 meses y 29 días en la comunidad quilombola en Ilha de Maré, Salvador/BA. Los resultados fueron: alergias $68,9 \%$ buscan el servicio de salud, diarrea $44,8 \%$ utilizan té, $58,6 \%$ en casos de fiebre utilizan la 
automedicación, $24 \%$ en episodios de vómito buscan el servicio de salud, $31,1 \%$ en casos de quemadura utilizan el enfriamiento. Aunque en algunos casos la comunidad busque el servicio de salud, la misma se encuentra ubicada a la larga distancia de éstos, lo que pueden ofrecer resolutividad. De esta forma, se hace necesario el estímulo de actividades educativas para que la comunidad tenga acceso a la salud de forma integral.

Palavras-clave: comunidad, primeros auxilios, niño, accidentes, salud.

Introdução

Comunidades quilombolas são grupos étnico-raciais segundo critérios de autoatribuição, com trajetória histórica própria, dotados de relações territoriais específicas e com presunção de ancestralidade negra relacionada com a resistência e opressão histórica sofrida [1].

A relação entre indivíduos de comunidades quilombolas e vulnerabilidade deve ser concebida a partir de um prisma multicausal, considerando-se o contexto histórico de formação dos quilombos e a ausência deste estrato populacional no que diz respeito à formulação de políticas públicas sociais e de saúde direcionadas especificamente para estas comunidades [2].

Neste sentido, estes indivíduos carecem de bens e serviços considerados essenciais à vida em sociedade. Logo, é preciso direcionar a política social e as medidas governamentais para a redução dos fatores de vulnerabilidade que ameaçam o bem-estar e a saúde das comunidades quilombolas [2].

Considerando-se a vulnerabilidade em saúde vivenciada pelos quilombolas, estes indivíduos carecem de serviços especializados de saúde em suas comunidades, não tendo suporte para cuidados essenciais como os de urgência e emergência [3].

As situações de urgência e emergência correspondem a um processo em que o risco está presente, exigindo o tratamento imediato do agravo diante da necessidade de manter as funções vitais e evitar incapacidade ou complicações graves [4]. Embora situações de urgência e emergência possam acontecer em qualquer grupo, as crianças são consideradas mais vulneráveis.

As crianças representam cerca de $20 \%$ dos atendimentos nas emergências por acidentes e as maiores proporções de acidentes ocorreram no ambiente domiciliar [5].

As situações de urgência e emergência pediátricas que mais ocorrem no Brasil são os acidentes domésticos como afogamentos, quedas, queimaduras e intoxicações. Além destes, outros agravos podem comprometer a higidez da criança, requerendo intervenções imediatas, caracterizadas como os primeiros socorros, visto que o atendimento ágil e eficaz pode evitar o agravamento do quadro e a ocorrência de óbito [5].

Nesse contexto, é fundamental que todos tenham acesso às informações sobre os principais acidentes, as formas de prevenções e ações diante das situações que exigem cuidados imediatos, a fim de minimizar complicações decorrentes de medidas intempestivas e inadequadas [6]. Essa ação é o que se chama de medidas de primeiros socorros.

Apesar de sua relevância no nosso país, o ensino de primeiros socorros ainda é pouco difundido. Prevalece o desconhecimento sobre o tema e o auxílio a vítimas em situações de urgência ou emergência apenas pelo impulso da solidariedade, sem treinamento adequado, o que pode causar danos irreparáveis [7].

Diante do exposto e compreendendo que é necessário identificar os conhecimentos para elaborar estratégias visando aprimorá-lo, o objetivo deste estudo foi identificar os conhecimentos, atitudes e práticas de pais e/ou responsáveis por crianças quilombolas acerca de medidas de primeiros socorros. A necessidade de difundir entre os indivíduos, especialmente aqueles que não dispõem de atendimentos de urgência/emergência na comunidade onde vivem, o conhecimento acerca do emprego de métodos de primeiros socorros, bem como as formas de prevenção de acidentes na infância, considerando que a maioria destes agravos são passíveis de prevenção, e que complicações podem ser minimizadas quando se sabe ofertar uma assistência caracterizada como primeiros socorros adequadamente.

\section{Material e métodos}

Trata-se de um estudo descritivo exploratório com abordagem quantitativa. Realizado na comunidade quilombola de Praia Grande, localizada em llha de Maré, na cidade de Salvador/BA. A llha de Maré localiza-se a cerca de $5 \mathrm{~km}$ de São Tomé de Paripe, subúrbio de Salvador. A llha de Maré possui uma população de 6.434 habitantes, distribuída em uma área de $13,79 \mathrm{~km}^{2}$ traduzindo-se numa densidade populacional de $384,80 \mathrm{hab} / \mathrm{km}^{2}$ [8]. Apresenta densidade 
demográfica menor que a média de Salvador que é $8.801,21$ (hab/km²) [8]. A Região Administrativa 18, onde se encontra as ilhas é a menos populosa de Salvador [9].

No âmbito municipal, Salvador é dividida em Regiões Administrativas (RA), a llha de Maré encontra-se na RA 18 - Ilhas. A Ilha de Maré possui 1.871 domicílios com 3,4 moradores, em média situados em pequenas localidades da borda litorânea denominadas: Itamoabo, Botelho, Santana, Neves, Praia Grande, Bananeiras, Maracanã, Porto dos Cavalos, Caquende e Martelo, Amêndoa, Ponta de Coroa, Ponta da Cacimba, Ponta de Areia, Engenho de Maré e Ponta do Ermitão [8]. Em Salvador existem quatro comunidades quilombolas certificadas pela Fundação Palmares: Praia Grande, Bananeiras, Porto dos Cavalos/Martelo e Ilha de Maré da qual $60 \%$ do território referem-se às comunidades quilombolas [10].

O local do estudo foi eleito por ter sido realizado anteriormente um estudo de mestrado intitulado "Itinerários Terapêuticos em Urgências e Emergências Pediátricas em uma comunidade quilombola", identificou-se a necessidade de um feedback para a população acerca dos principais resultados, optando-se pela continuidade do estudo, que dessa vez buscou identificar o conhecimento, atitudes e práticas de pais e/ou responsáveis sobre primeiros socorros em pediatria.

A comunidade quilombola de Praia Grande também foi escolhida por conta de seu contexto de vulnerabilidade social, assim como pela mesma não possuir nenhum serviço de atendimento de urgência e emergência, o que traz riscos às crianças da comunidade, quando pais e responsáveis não sabem o que fazer diante de tais situações.

Participaram do estudo pais e/ou responsáveis por crianças quilombolas de 0 a 11 anos, 11 meses e 29 dias, considerando que, de acordo com o Estatuto da Criança e do Adolescente (ECA), em seu Art. $2^{\circ}$, considera-se criança a pessoa até doze anos de idade incompletos [11].

Os critérios de seleção foram: residir em Praia Grande no momento do estudo, ter idade igual ou superior a 18 anos, não possuir restrição cognitiva ou verbal que impedisse 0 desenvolvimento da entrevista, conviverem e/ou cuidarem de crianças menores de 12 anos e aceitar participar da pesquisa de forma voluntária.

Inicialmente, foi realizada uma visita domiciliar a 29 pais e/ou responsáveis de crianças aleatórios da comunidade de Praia Grande para aplicar um instrumento de entrevista com sete perguntas acerca de seus conhecimentos, atitudes e práticas diante de situações de urgência e emergência com crianças menores de 12 anos a fim de compreender o conhecimento dos participantes acerca de primeiros socorros em pediatria.

As variáveis contempladas no instrumento foram: crise alérgica, diarreia, febre, queimadura e vômito. Os temas abordados foram propostos pela comunidade em um estudo anterior (Itinerários terapêuticos em urgências e emergências pediátricas em uma comunidade quilombola) onde foram identificados os principais agravos que acometem as crianças da referida comunidade.

Os dados coletados foram organizados e analisados utilizando-se o programa Statistical Package for the Social Sciences (SPSS), Windows, versão 12.0. A análise foi realizada através de estatística descritiva utilizando medidas de frequência. Os dados analisados foram organizados em tabelas e apresentados sob a forma de números e percentuais.

Este estudo foi aprovado pelo Comitê de Ética em Pesquisa de uma universidade pública, sob o protocolo de no 420.096. Em todas as etapas da pesquisa foram seguidas as recomendações da Resolução 466/2012, do Conselho Nacional de Saúde (CNS) que regulamenta a pesquisa envolvendo seres humanos [12].

Todos os participantes que colaboraram para esta pesquisa foram informados acerca dos objetivos e importância deste estudo, bem como dos riscos a que estariam sujeitos, traduzidos na possibilidade de aceitarem participar do estudo, foram explicados sobre sua participação, que ocorreu mediante a leitura e assinatura do Termo de Consentimento Livre e Esclarecido. Sendo asseguradas a confiabilidade, o anonimato e a privacidade das informações coletadas.

Resultados

Foram entrevistados 29 participantes responsáveis por crianças de 0 a 11 anos, 11 meses e 29 dias residentes em Praia Grande, Salvador/BA.

A tabela I mostra o que os pais/responsáveis fazem acerca do episódio de intoxicações. 
Tabela I - Conhecimento de pais e responsáveis sobre medidas de primeiros socorros diante de crise alérgica, Praia Grande, Salvador/BA 2016. $N=29$.

\begin{tabular}{lll}
\hline $\begin{array}{l}\text { O que se deve fazer quando a criança } \\
\text { apresenta reação alérgica? }\end{array}$ & No. $^{*}$ & $\%^{\star *}$ \\
\hline Levar ao serviço de saúde & 20 & 68.9 \\
Dar chá & 1 & 3,4 \\
Automedicação & 2 & 7,0 \\
Outros procedimentos & 5 & 17,2 \\
Não sabe & 1 & 3,5 \\
\hline
\end{tabular}

${ }^{*}$ No, número, \%**, percentual.

Quando indagados sobre o que fazem em situação de diarreia, $44,8 \%$ dos participantes, ou seja, 13 deles referiram dar chá às crianças como mostra a tabela II.

Tabela II - Conhecimento de pais e responsáveis sobre medidas de primeiros socorros diante de episódios de diarreia, Praia Grande, Salvador/BA, 2016. N=29.

\begin{tabular}{lll}
\hline $\begin{array}{l}\text { O que se deve fazer quando a criança } \\
\text { apresenta diarreia? }\end{array}$ & No. $^{*}$ & $\%^{\star \star}$ \\
\hline Levar ao serviço de saúde & 7 & 24,1 \\
Automedicação & 2 & 7,0 \\
Dar soro caseiro & 7 & 24,1 \\
Dar chá & 13 & 44,8 \\
\hline
\end{tabular}

*No, número, \%**, percentual.

Em caso de crianças com febre alta, $58,6 \%$ dos entrevistados, ou seja, 17 dos 29 participantes confirmam o uso de automedicação como tentativa de melhora do quadro como pode ser visto na tabela III.

Tabela III - Conhecimento de pais e responsáveis sobre medidas de primeiros socorros diante episódios de febre alta Praia Grande, Salvador/BA, 2016. $N=29$.

\begin{tabular}{lll}
\hline $\begin{array}{l}\text { O que se deve fazer quando a criança } \\
\text { apresenta febre alta? }\end{array}$ & No. $^{*}$ & $\%^{* *}$ \\
\hline Levar ao serviço de saúde & 5 & 17,3 \\
Automedicação & 17 & 58,6 \\
Resfriamento & 5 & 17,3 \\
Outros procedimentos & 1 & 3,4 \\
Não sabe & 1 & 3,4 \\
\hline
\end{tabular}

${ }^{*}$ No, número, \%* ${ }^{\star *}$ percentual.

A respeito de episódios de vômitos, $24 \%$ dos participantes responderam que levariam ao serviço de saúde, enquanto houve a frequência de $20,6 \%$ que referiram buscar o serviço de saúde. Tabela IV.

Tabela IV - Conhecimento de pais e responsáveis sobre medidas de primeiros socorros diante de episódios de vômito, Praia Grande, Salvador, Bahia, 2016. N=29.

\begin{tabular}{lll}
\hline $\begin{array}{l}\text { O que se deve fazer quando a criança } \\
\text { está vomitando? }\end{array}$ & No. $^{*}$ & $\%^{* *}$ \\
\hline Levar ao serviço de saúde & 7 & 24,0 \\
Automedicação & 5 & 17,3 \\
Dar chá & 6 & 20,6 \\
Dar soro caseiro & 2 & 7,0 \\
Outros procedimentos & 4 & 13,8 \\
Não sabe & 5 & 17,3 \\
\hline
\end{tabular}

${ }^{*}$ No, número, $\%{ }^{* *}$, percentual.

Em caso de queimaduras, $31,1 \%$ dos participantes relataram o uso de resfriamento à criança vítima, enquanto $30,9 \%$ levariam ao serviço de saúde como pode ser visto na tabela $\mathrm{V}$. 
Tabela V - Conhecimento de pais e responsáveis sobre medidas de primeiros socorros diante de queimaduras, Praia Grande, Salvador/BA, 2016. $N=29$.

\begin{tabular}{lll}
\hline $\begin{array}{l}\text { O que se deve fazer quando a criança se } \\
\text { queima? }\end{array}$ & $\mathbf{N}^{*}$ & $\%^{\star *}$ \\
\hline Levar ao serviço de saúde & 9 & 30,9 \\
Automedicação & 2 & 7,0 \\
Resfriamento & 9 & 31,1 \\
Outros procedimentos & 6 & 20,7 \\
Não sabe & 3 & 10,3 \\
\hline
\end{tabular}

*No, número, \%**, percentual.

Discussão

Neste estudo foi identificado que a maioria dos pais e/ou responsáveis de crianças de 0 a 11 anos buscam por serviço de saúde no continente quando ocorre alguma das situações de urgência e emergência por não saberem como atuar de forma eficiente e imediata durante os quadros.

Um estudo afirma que o ensino de primeiros socorros deveria ser mais acessível e disseminado para as pessoas leigas e população em geral. Aprender sobre primeiros socorros ajudaria os indivíduos a atuar com maior segurança caso ocorresse uma situação de emergência na comunidade. Assim, tendo maiores conhecimentos diminuiria os agravos à saúde da vítima [6].

Outro estudo cita ser ideal que toda a população consiga saber ao menos os princípios básicos dos primeiros socorros, uma vez que nosso cotidiano é cheio de acidentes e situações vulneráveis, quando a assistência e o uso de manobras de primeiros socorros se fazem necessários [13]. Porém, o que identificamos e vivemos é ao contrário, uma população com déficit de informação sobre primeiros socorros, principalmente quando se refere a uma faixa etária vulnerável, as crianças.

Em caso de alergias, 68,9\% (conforme tabela I) dos participantes referiram a busca por serviço de saúde como primeira e única medida adotada. Considerando o contexto de acesso e transporte, a falta de conhecimento de medidas de primeiros socorros pode representar um risco à vida, pois os sinais e sintomas de crises alérgicas podem evoluir rapidamente. 17,2\% (conforme tabela I) potencializam ingestão de líquidos, e observam último alimento ingerido pela criança, visando parar o uso do mesmo na tentativa de minimizar o quadro.

Além dos problemas respiratórios, o mapa de justiça ambiental acrescenta que a comunidade também tem sofrido com problemas respiratórios, de visão e outras doenças ligadas às substâncias químicas, como amônia, xileno, soda cáustica e nafta petroquímica [14].

Os três pescados (chumbinho, siri e sururu) mais consumidos na llha de Maré apresentaram altos níveis de contaminação por metais pesados, entre eles o chumbo, cádmio e o mercúrio. O consumo dos pescados contaminados está associado ao risco de doenças graves como o câncer. Em crianças, a absorção dessas substâncias é maior [10].

Quando se tratou de diarreia $44,8 \%$ (conforme tabela III) dos pais e/ou responsáveis, referiram 0 uso de chás como medida inicial para melhora de diarreia nas crianças. $O$ conhecimento popular pode ser evidenciado na área da saúde uma vez que os indivíduos buscam formas de tratamento e prevenção de doenças vinculadas a crenças e tradições.

Dentre os cuidados ao RN e crianças que são baseados em saberes popular estão o uso de chás, a prática do benzer e a utilização de diversas substâncias [15]. Dentre os chás citados pelos participantes destacaram-se: chá de erva doce, chá de folha de araçá, folha da casca de banana, entre outros.

Conforme tabela III, $24,1 \%$ dos participantes utilizam como medida inicial o soro caseiro. As doenças diarreicas agudas são importantes causas de morbimortalidade no Brasil e no mundo. Anualmente, cerca de 2,5 bilhões de casos de diarreia ocorrem em crianças menores de cinco anos de idade. Os fatores de risco associados à diarreia são múltiplos, incluindo aspectos socioeconômicos, políticos, demográficos, sanitários, ambientais e culturais. Apesar dos inúmeros fatores que condicionam a diarreia, a incidência da doença poderia ser minimizada a partir de práticas cotidianas de saúde, sobretudo aquelas relacionadas aos hábitos higiênicos [16].

O Ministério da Saúde distribui gratuitamente nos postos do país e nas unidades da rede Farmácia Popular o soro de reposição oral, que concentra 3,5 gramas de sal e 20 gramas de açúcar por litro de água. Atualmente, o soro caseiro só é recomendado em casos de emergência, 
pois os pais costumam fazer superdose nesse preparo [17]. Muitos pais e responsáveis declaram o uso do soro caseiro como primeira medida, mas expressaram dificuldade em saber seu preparo ideal.

A maneira apropriada de usar o soro de reidratação oral está descrita na Caderneta de Saúde da Criança, entregue aos pais assim que saem da maternidade pública, porém como se trata de uma comunidade com um déficit em nível de conhecimento e escolaridade a maioria desconhece a informação.

Conforme tabela III, $7,0 \%$ dos participantes referiram uso de remédios caseiros e automedicação. O uso de medicamentos de forma incorreta pode acarretar o agravamento de uma doença, uma vez que a utilização inadequada pode esconder determinados sintomas.

A automedicação nem sempre é adequada e pode ter efeitos colaterais desagradáveis, além de 'mascarar' uma doença mais grave, que poderia ser diagnosticada caso os remédios não tivessem sido ingeridos incorretamente.

No que se questionou sobre a febre alta, $58,6 \%$ (conforme tabela IV) dos entrevistados referiram a automedicação como primeira medida quando ocorre a elevação de temperatura na criança. Posteriormente aparece o resfriamento e a busca por serviço de saúde com 17,3\% (conforme tabela IV) na tentativa de controle da febre.

O consumo de medicamentos pode ser considerado um indicador indireto de qualidade dos serviços de saúde, crianças e adolescentes representam um grupo fortemente predisposto ao uso irracional de medicamentos com e sem controle médico, devido ao conhecimento prévio e saber popular de seus pais e/ou responsáveis [18].

Existe uma predominância da administração dos medicamentos não prescritos às crianças pelas mães. Tal atitude tem sido atribuída a papéis sociais tradicionalmente delegados às mães, dentre eles, o de prover a saúde da família. As principais situações de saúde que motivam a automedicação são tosse, resfriado comum, gripe, congestão nasal ou broncoespasmo (17,2\%); febre (15\%); cefaleia (14\%); diarreia, "má digestão" e cólica abdominal (9\%) [19].

Fatores econômicos, políticos e culturais têm contribuído para o crescimento e a difusão da automedicação na sociedade. Acredito que fatores se relacionam, dentre outros, a uma grande disponibilidade de produtos; simbolização da saúde que o medicamento pode representar; publicidade irresponsável; facilidade em comprar medicamentos; qualidade da assistência à saúde; dificuldade de acesso aos serviços de saúde como é o caso da comunidade quilombola em questão.

Náuseas e vômitos são sintomas muito comuns na população pediátrica. Seja em um pronto-socorro ou ambulatório, é motivo frequente para pais e cuidadores procurarem atendimento médico. Consequentemente, tem um impacto significativo nos custos com saúde [20].

O vômito é definido como a expulsão oral vigorosa do conteúdo gástrico ou intestinal associada à pressão intra-abdominal elevada. A regurgitação oral refere-se à êmese do conteúdo alimentar, feita sem esforço, geralmente pós-prandial [19]. Uma anamnese completa e exame físico são essenciais e fornecem indícios para o diagnóstico e tratamento adequados. Os testes diagnósticos devem ser orientados pelo quadro clínico.

Considerando as queimaduras, conforme tabela $\mathrm{VI}, 31,1 \%$ dos participantes utilizam o resfriamento como primeira medida de intervenção após queimaduras em crianças. Enquanto, $30,9 \%$ referiram procurar serviço de saúde e outros $20,7 \%$ realizam outros procedimentos (lavam com água e sabão, colocam pasta, pomadas, remédios caseiros).

As lesões por queimadura constituem uma importante causa acidental de morbimortalidade em todo o mundo, com grande frequência entre as crianças, sendo as escaldaduras (produzidas por substâncias quentes ou fontes de calor) as mais comuns, além das queimaduras químicas, elétricas e radioativas. A queimadura é apontada em vários estudos entre as causas acidentais mais frequentes entre crianças e adolescentes [21].

Estudos mostram que um dos primeiros e mais utilizados tratamentos de queimaduras é o resfriamento da superfície atingida. O resfriamento tem potenciais benéficos, tais como proporcionar alívio da dor, menor formação de edemas, redução da taxa de infecção, assim como do aprofundamento da lesão, cicatrização mais rápida, menor necessidade de enxertia, diminuição das cicatrizes e da mortalidade [22]. Embora o resfriamento deva ter início o mais rapidamente possível, sua realização mesmo que tardia pode ser benéfica.

O estudo reafirma a situação de vulnerabilidade da população negra, em especial a quilombola de llha de Maré, na questão do acesso e da utilização dos serviços de saúde. A procura por atendimento médico demonstra ser após tentativa de saber popular, revelando assim, 
a insatisfação e angústia com a dificuldade de acesso dos quilombolas aos serviços de saúde, em especial em situações de urgência e emergência envolvendo crianças.

O presente trabalho buscou identificar quais as medidas de primeiros socorros prestados a crianças por pais e/ou responsáveis diante de situações de urgência e emergência, visto que se trata de uma comunidade vulnerável, onde o acesso ao serviço de saúde é limitado.

Diante do exposto, percebemos que existe sempre a primeira alternativa de pais e/ou responsáveis em atuarem com as crianças, geralmente muitos utilizam o saber popular, como alternativa de resolução aos agravos estudados, ou fica vulnerável à busca por atendimento médico no continente, o que na maioria das vezes não é de forma imediata, podendo ocorrer complicações à saúde da criança.

De acordo com os resultados alcançados, foi observado que o saber popular na atuação de pais e responsáveis com crianças em situações de agravos à saúde é transmitido de geração em geração, tendo seus valores econômicos, sociais e culturais preservados, podendo o conhecimento formal agregar de forma positiva para a comunidade. Apesar das peculiaridades do conhecimento científico e do conhecimento popular, notam-se certas semelhanças entre eles, sendo de características distintas e originalmente parecidas.

Os pais e/ou responsáveis são os melhores fornecedores de informações acerca das dúvidas e dificuldades, experiências e necessidades. Por isso, este estudo foi de grande valia para refletirmos sobre o que têm sido feito com crianças em situações de agravo à saúde das mesmas e desta forma pudermos traçar estratégias para colaborar e ampliar o conhecimento já existente ao saber formal da temática.

O conhecimento da comunidade sobre as temáticas coincide com as suas atitudes e práticas frente às situações de urgência e emergência. $O$ que elas sabem é reafirmado com o que elas fazem diante dos agravos. Utilizam saberes e fazeres que aparecem como aqueles fundantes de sua tradicionalidade, como: rezas, remédios caseiros, banho de ervas, chás, isso muitas vezes, para eles signifique a sobrevivência de filhos, netos, bisnetos, sobrinhos.

1. Ministério da Saúde (BR). Condições de saúde da população brasileira: resultados principais. Brasília: Ministério da Saúde; 2003.

2. Silva MLM, Rangel RF, Zanatta FB, Backes DS, Costenaro RGS, Piovesan C, et al. Indicadores de risco associados à qualidade de vida de escolares de uma comunidade vulnerável do sul do Brasil [Internet]. Disciplinarum Scientia. Série Ciências da Saúde 2013;14(1):163-71.

3. Siqueira SMC. Itinerários terapêuticos em urgências e emergências pediátricas em uma comunidade quilombola [Dissertação]. Salvador: Escola de Enfermagem, Universidade Federal da Bahia; 2014.

4. Melo EM. Podemos prevenir a violência. Organização Pan-Americana de Saúde. Serie: Promoção de Saúde e Prevenção da Violência. Brasília: OPS; 2010.

5. Ministério da Saúde (BR). A saúde da população negra e o SUS: ações afirmativas para avançar na equidade. Brasília: Ministério da Saúde; 2005.

6. Nardino J, Rossato MB, Bisogno SBC, Guth EJ. Atividades Educativas em Primeiros Socorros. Rev Contexto e Saúde 2012;12(23). https://doi.org/10.21527/21767114.2012.23.88-92

7. Pergola AM, Araujo IEM. O leigo em situação de emergência. Rev Esc Enferm USP 2008;42(4):769-76. https://doi.org/10.1590/S0080-62342008000400021

8. Ministério do Planejamento, Orçamento e Gestão. Instituto Brasileiro de Geografia e Estatística (IBGE). Censo demográfico 2010: características da população e dos domicílios: resultados do universo [Internet]. 2011 [citado 2016 Fev 3]. http://biblioteca.ibge.gov.br/visualizacao/periodicos/93/cd_2010_caracteristicas_popula cao_domicilios.pdf.

9. SIM. Sistema de Informações Municipais de Salvador. Dados do Censo do IBGE de 2010 [citado 2014 Nov 18]; Disponível em: http://www.sim.salvador.ba.gov.br/indicadores/ 
10. Radis. Águas contaminadas na llha de Maré. 129 • jun/ 2013 [citado 2014 Nov 22]. http://www6.ensp.fiocruz.br/radis/sites/default/files/copia de radis 129 23maio2013.pd f

11. Ministério da Saúde (BR). Estatuto da criança e do adolescente: Lei federal no 8069, de 13 de julho de 1990. Rio de Janeiro: Ministério da Saúde; 2002.

12. Ministério da Saúde (BR). Conselho Nacional de Saúde. Resolução n ${ }^{\circ} 466$, de 12 de dezembro de 2012. Aprova normas regulamentadoras de pesquisas envolvendo seres humanos. Brasília: Diário Oficial da União; 2013.

13. Souza VRFP. Conceição das Crioulas, Salgueiro (PE). In: O’Dwyer EC. (Org.). Quilombos: identidade étnica e territorialidade. Rio de Janeiro: FGV; 2002. p. 109-140.

14. Fuchs $\mathrm{KM}$. Estudo epidemiológico de queixas relacionadas à rinite em marisqueiras da Ilha de Maré [TCC]. Salvador: Universidade Federal da Bahia; 2013.

15. Costa ACPJ, Bandeira LPL, Araújo MFM de, Gubert FA, Rebouças CBA, et al. Saberes populares no cuidado ao recém-nascido com enfoque na promoção da saúde. Rev Pesq: Cuid Fundam online 2013;5(2):3626-35.

16. Farthing M, Salam MA, Lindberg G, Dite P, Khalif I, Salazar-Lindo E, et al. Acute diarrhea in adults and children: a global perspective. J Clin Gastroenterol 2013;47:1220. https://doi.org/10.1097/MCG.0b013e31826df662

17. Pour TR, Koyfman A, Rumyar MS. Emergence centre management of pediatric diarrhea: an overview. Afr J Emerg Med 2013;3:75-82. https://doi.org/10.1016/..afjem.2012.06.004

18. Arrais PSD, Fernandes MEP, Pizzo T da SD, Ramos LR, Mengue SS. Prevalência da automedicação no Brasil e fatores associados. Rev Saúde Pública 2016;50 (supl. 2):1s11s. https://doi.org/10.5123/s1679-49742017000200009

19. Prado TF, Carmo PC, PJS. Automedicação em crianças de zero a cinco anos: fármacos administrados, conhecimentos, motivos e justificativas. Escola Anna Nery Revista de Enfermagem 2013;17(Pt 2):291-7. https://doi.org/10.1590/S141481452013000200013

20. Fedorowicz Z, Jagannath V, Carter B. Antiemetics for reducing vomiting related to acute gastroenteritis in children and adolescents. Cochrane Database Syst Rev 2011;(9). https://doi.org/10.1002/14651858.CD005506.pub5

21. Campos JA, Paes CEN, Blank D, Costa DM, Pfeiffer L, Waksman RD. Manual de segurança da criança e do adolescente. São Paulo: Sociedade Brasileira de Pediatria/Nestle Nutriçao; 2004.

22. Gathas AZ, Djaleta DG, Noviello DS, Thomaz MCA, Arçari DP. Atendimento do enfermeiro ao paciente queimado. Saúde em Foco 2011;5(8):1-20. 